\title{
Theoretical cross sections of tantalum on neutron induced reactions
}

\author{
Tarik Siddik \\ Salahaddin University-College of Basic Education-Department of General Science, Erbil, Iraq
}

\begin{abstract}
Neutron-induced cross-sections for the stable isotope ${ }^{181} \mathrm{Ta}$, in the energy region up to $20 \mathrm{MeV}$ have been calculated. Statistical model calculations, based on the Hauser-Feshbach formalism, have been carried out using the TALYS-1.0 and were compared with available experimental data in the literature and with ENDF/B-VII, T = $300 \mathrm{~K}$; JENDL-3.3, T = $300 \mathrm{~K}$ and JEFF-3.1, T = $300 \mathrm{~K}$ evaluated libraries.
\end{abstract}

\section{Introduction}

With the advent of fast computers, software that simulates nuclear reactions is able to play an increasingly important role in nuclear data. It is possible to provide an exact computational scheme for sophisticated nuclear models, not only new ones but also those that have been lying on the shelf for decades and only now become amenable for numerical implementation. Large scale comparisons with measurements are within reach. TALYS is a nuclear reaction program created at NRG Petten, the Netherlands, and CEA Bruyères-le-Châtel,France. The purpose of TALYS is to simulate nuclear reactions that involve neutrons, photons, protons, deuterons, tritons, ${ }^{3} \mathrm{He}$, and $\alpha$ particles in the $1 \mathrm{keV}$ to $200 \mathrm{MeV}$ energy range. Predicted quantities include integrated, single and double-differential cross sections, for both the continuum and discrete states, residue production and fission cross sections, $\gamma$-ray production cross sections, etc. Nuclear cross section data can be obtained by three different ways on the basis of experimental measurement, theoretical model calculations and evaluation data files. Experimental cross section data in the range $15-20 \mathrm{MeV}$ are not available for all neutron induced reactions on Tantalum isotopes however, there is only a limited number of data for at the energy range $<15 \mathrm{MeV}$ [1-4].

\section{Calculation Methods}

The high-energy end of the ejectile spectra are described by preequilibrium emission, which takes place after the first stage of the reaction but long before statistical equilibrium of the compound nucleus is attained. It is imagined that the incident particle creates step by step more complex states in the compound system and gradually loses its memory of the initial energy and direction. The default preequilibrium model of TALYS is the two-component exciton model [5,6]. We recall the basic formula of [5] for the exciton model cross section,

$$
\begin{aligned}
\frac{d \sigma_{k}^{\mathrm{EM}}}{d E_{k}}= & \sigma^{\mathrm{CF}} \sum_{p_{\pi}=p_{\pi}^{0}}^{p_{\pi}^{\mathrm{eq}}} \sum_{p_{v}=p_{v}^{0}}^{p_{v}^{\mathrm{q}}} w_{k}\left(p_{\pi}, h_{\pi}, p_{v}, h_{v}, E_{k}\right) \\
& \times S_{\mathrm{pre}}\left(p_{\pi}, h_{\pi}, p_{v}, h_{v}\right),
\end{aligned}
$$

where $\mathrm{p} \pi(\mathrm{p} v)$ is the proton (neutron) particle number and $h \pi(h v)$ the proton (neutron) hole number, $\sigma^{\mathrm{CF}}$ is the compound formation cross section, and $\mathrm{S}_{\text {pre }}$ is the timeintegrated strength which determines how long the system remains in a certain exciton configuration. The initial proton and neutron particle numbers are denoted $\mathrm{p}_{\pi}^{0}=\mathrm{Z}_{\mathrm{p}}$ and $\mathrm{p}_{v}^{0}=\mathrm{N}_{\mathrm{p}}$ with $\mathrm{Z}_{\mathrm{p}}\left(\mathrm{N}_{\mathrm{p}}\right)$ being the proton (neutron) number of the projectile. In general, $\mathrm{h}_{\pi}=\mathrm{p}_{\pi}$ and $\mathrm{h}_{\mathrm{v}}=\mathrm{p}_{\mathrm{v}}-\mathrm{p}_{\mathrm{v}}^{\mathrm{o}}$ so that the initial hole numbers are zero, i.e., $h_{\pi}^{0}=h_{v}^{0}=0$ for primary preequilibrium emission. The preequilibrium part is calculated by Eq. (1), using $\mathrm{p}_{\pi}^{e \mathrm{q}}=\mathrm{p}_{\mathrm{v}}^{\mathrm{eq}}=6$, whereas the remainder of the reaction flux is distributed through the Hauser-Feshbach model. In addition, the never-come-back approximation is adopted. The emission rate wk for ejectile $\mathrm{k}$ with spin sk is given by

$$
\begin{gathered}
w_{k}\left(p_{\pi}, h_{\pi}, p_{v}, h_{v}, E_{k}\right)=\frac{2 s_{k}+1}{\pi^{2} \hbar^{3}} \mu_{k} E_{k} \sigma_{k, \text { inv }}\left(E_{k}\right) \\
\times \frac{\omega\left(p_{\pi}-Z_{k}, h_{\pi}, p_{v}-N_{k}, h_{v}, E_{x}\right)}{\omega\left(p_{\pi}, h_{\pi}, p_{v}, h_{v}, E^{\text {tot }}\right)}
\end{gathered}
$$

where $\sigma_{\mathrm{k}, \text { inv }}(\mathrm{Ek})$ is the inverse reaction cross section as calculated from the optical model, and $\omega$ is the twocomponent particle-hole state density. The full reaction dynamics that leads to Eq. (1) is described in Refs. $[5,6]$. We here restrict ourselves to the formulas given above since they contain the model- and parameterdependent quantities. The expression for $\mathrm{S}_{\text {pre }}$ contains the adjustable transition matrix element M2 for each possible transition between neutron-proton exciton 
configurations. A proton-neutron ratio of 1.6 for the squared internal transition matrix elements was adopted to give the best overall agreement with experiment, i.e., $\mathrm{M}_{\pi v}^{2}=\mathrm{M}_{v \pi}^{2}=1.6 \mathrm{M}_{v \pi}^{2}=1.6 \mathrm{M}^{2}$. Partial level density parameters $g \pi=Z / 17$ and $g v=N / 17$ were used in the equidistant spacing model for the partial level densities. Finally, an effective surface interaction well depth $\mathrm{V}=12$ $\mathrm{MeV}$ [5] was used. At incident energies above several tens of $\mathrm{MeV}$, the residual nuclides formed after binary emission may have so large an excitation energy that the presence of additional fast particles inside the nucleus becomes possible. The latter can be imagined as strongly excited particle-hole pairs resulting from the first binary interaction with the projectile. The residual system is then clearly non-equilibrated, and the excited particle that is high in the continuum may, in addition to the first emitted particle, be emitted on a short time scale. This so-called multiple preequilibrium emission forms an alternative theoretical picture of the intranuclear cascade process, whereby the exact location and momentum of the particles are not followed, but instead the total energy of the system and the number of particle-hole excitations (exciton number). In actual calculations, the particle-hole configuration of the residual nucleus after emission of the ejectile, is reentered as an initial condition in Eq. (1). When looping over all possible residual configurations, the multiple preequilibrium contribution is obtained [7-20]. In TALYS, multiple preequilibrium emission is followed up to arbitrary order; though for $96 \mathrm{MeV}$, only the secondary preequilibrium emission is significant. It is well known that semiclassical models, such as the exciton model, have had some problems in describing angular distributions (essentially because the model is based on a compound like concept instead of a direct one). Therefore, as mentioned previously, the doubledifferential cross sections are obtained from the calculated energy spectra using the Kalbach systematics [11]. To account for the evaporation peaks in the charged-particle spectra, multiple compound emission was treated with the Hauser-Feshbach model. In this scheme, all reaction chains are followed until all emission channels are closed. The Ignatyuk model [12] has been adopted for the total level density to account for the damping of shell effects at high excitation energies. For preequilibrium reactions involving deuterons, tritons, ${ }^{3} \mathrm{He}$ and $\alpha$ particles, a statistical contribution from the exciton model is automatically calculated with the formalism described above. However, it is well known that for nuclear reactions involving projectiles and ejectiles with different particle numbers, mechanisms such as stripping, pickup, and knockout play an important role, and these direct like reactions to the continuum are not covered by the exciton model. Therefore, Kalbach has developed a phenomenological contribution for these mechanisms [11], which is included in TALYS. The advantages over the older method include a better consideration of the available phase space through normalized particle-hole state densities and a better empirical determination of the pickup, stripping, knockout strength parameters, enabled by the more extensive experimental database that is now available. It has recently been shown that for medium and heavy nuclides this method gives a considerable improvement over the older methods. The latter seemed to consistently under predict neutroninduced reaction cross sections involving pickup of one or a few nucleons.
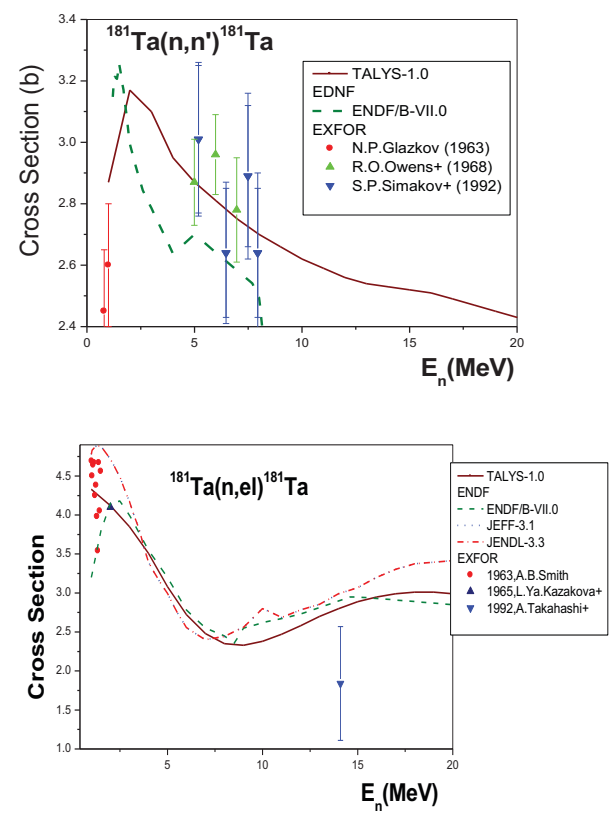

Fig. 1. The comparison of calculated excitation function using TALYS of ${ }^{181} \mathrm{Ta}\left(\mathrm{n}, \mathrm{n}^{\prime}\right) \&{ }^{181} \mathrm{Ta}(\mathrm{n}, \mathrm{el})$ reaction with available experimental values and evaluated nuclear data files ENDF/BVI . The values reported in Ref. (21)
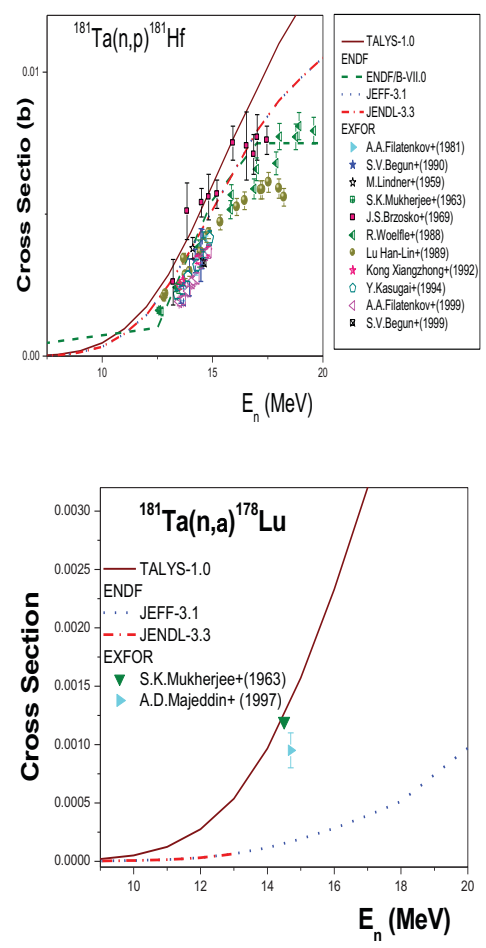

Fig. 2. The comparison of calculated excitation function using TALYS of ${ }^{181} \mathrm{Ta}(\mathrm{n}, \mathrm{p})$ and ${ }^{181} \mathrm{Ta}(\mathrm{n}, \alpha)$ reaction with available experimental values and evaluated nuclear data files ENDF/BVI, JENDL-3.3 and JEFF3.1.The values reported in Ref. (21) 

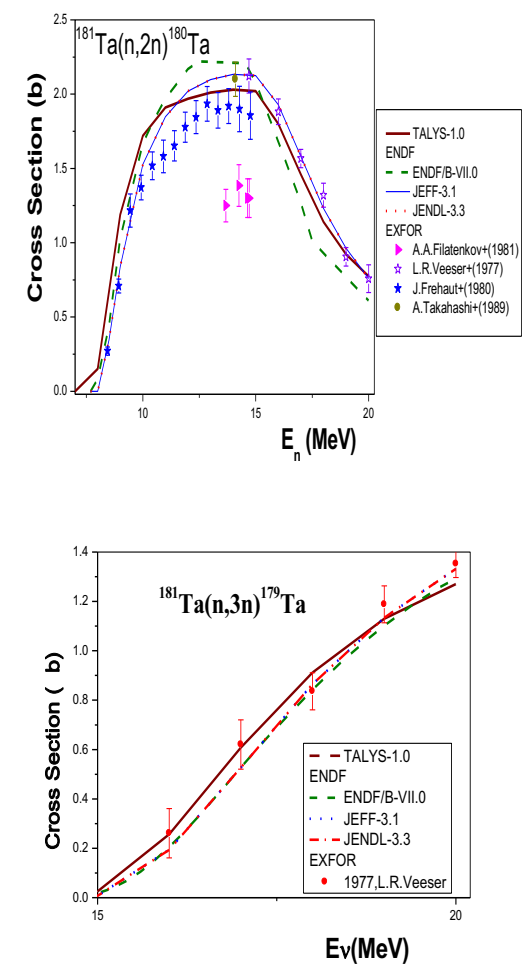

Fig. 3. The comparison of calculated excitation function using TALYS of ${ }^{181} \mathrm{Ta}(\mathrm{n}, 2 \mathrm{n}) \&{ }^{181} \mathrm{Ta}(\mathrm{n}, 3 \mathrm{n})$ reaction with available experimental values and evaluated nuclear data files ENDF/BVI, JENDL-3.3 and JEFF3.1. The values reported in Ref. (21)

\section{Calculations and analysis}

In Figs. $\quad 1-3,{ }^{181} \mathrm{Ta}(\mathrm{n}, \mathrm{inl}),{ }^{181} \mathrm{Ta}(\mathrm{n}, \mathrm{el}), \quad{ }^{181} \mathrm{Ta}(\mathrm{n}, \mathrm{p})$, ${ }^{181} \mathrm{Ta}(\mathrm{n}, \alpha),{ }^{181} \mathrm{Ta}(\mathrm{n}, 2 \mathrm{n}), \quad{ }^{181} \mathrm{Ta}(\mathrm{n}, 3 \mathrm{n}) \quad$ reaction $\quad$ cross sections have been shown, respectively. The new calculations on the excitation functions of present paper have been carried out up to $20 \mathrm{MeV}$ incident neutron energy. The results of our calculations using TALYS1.0 code are compared with available experimental data in the EXFOR/CSISRS (2008) and with ENDF/B-VII.0 $\mathrm{T}=300 \mathrm{~K}, \mathrm{JENDL}-3.3 \mathrm{~T}=300 \mathrm{~K}$ and JEFF3.1, $\mathrm{T}=300 \mathrm{k}$ evaluated data libraries. A reasonable agreement with experimental and theoretical excitation functions was obtained. The results can be summarized and conclude as follows:

\section{1 (n,inl) Reactions}

There are only a limited number of experimental cross section data for ${ }^{181} \mathrm{Ta}(\mathrm{n}, \mathrm{inl})$ reaction in the energy range up to $20 \mathrm{MeV}$ Fig. 1. The calculation of TALYS-1.0 code of ${ }^{181} \mathrm{Ta}(\mathrm{n}, \mathrm{inl})$ reaction are in good agreement with the measurements between 5 and $8 \mathrm{MeV}$.

\section{2 (n,el) Reactions}

There are only a limited number of experimental cross section data for ${ }^{181} \mathrm{Ta}(\mathrm{n}, \mathrm{inl})$ reaction in the energy range up to $20 \mathrm{MeV}$ Fig. 1.

\section{$3.3(n, p)$ Reactions}

High discrepancies are observed in experimental data for ${ }^{181} \mathrm{Ta}(\mathrm{n}, \mathrm{p})$ The calculation of TALYS-1.0 code of ${ }^{181} \mathrm{Ta}(\mathrm{n}, \mathrm{p})$ reaction are in good agreement with the measurements between 12 and $17 \mathrm{MeV}$ Fig. 2. The evaluated nuclear data files JEFF-3.1 and JENDL-3.3 Show important discrepancies in energies $<16 \mathrm{MeV}$.

\section{$3.4(n, \alpha)$ Reactions}

There are only two experiment value for ${ }^{181} \mathrm{Ta}(\mathrm{n}, \alpha)$ Fig. 2. TALYS-1.0 has been used for ${ }^{181} \mathrm{Ta}(\mathrm{n}, \alpha)$ reaction.

\section{$3.5(n, 2 n)$ Reactions}

The calculation of TALYS-1.0 code of ${ }^{181} \mathrm{Ta}(\mathrm{n}, 2 \mathrm{n})$ reaction are in good agreement with the measurements between 12 and $20 \mathrm{MeV}$ Fig. 3. ENDEF/B-VI, JENDL3.3 and JEFF-3.1 files are in agreement with each other.

\section{$3.6(n, 3 n)$ reactions}

The calculation of TALYS-1.0 code of ${ }^{181} \mathrm{Ta}(\mathrm{n}, 3 \mathrm{n})$ reaction are in good agreement with the experimental data Fig. 3. ENDEF/B-VI, JENDL-3.3 and JEFF-3.1 files are in agreement with each other.

\section{References}

1. T. Siddik, J. Fusion Energ., 32, 164, (2013).

2. E. Tel, J. Fusion Energ., 29, 332, (2010).

3. T. Siddik, J. Fusion Energ., 34, (2015).

4. T. Siddik, $30^{\text {th }}$ International Physics Congress/ Istanbul/Turkey (2013).

5. A.J. Koning, M.C. Duijvestijn, Nucl. Phys. A744, 15 (2004).

6. C. Kalbach, Phys. Rev., C33, 818 (1986).

7. T. Siddik, J. Advanced Physics, 5, 1, (2016).

8. E. Tel, et al., Acta Phys. Slov. 54 (2), 191 (2004).

9. A.J. Koning, J.-P. Delaroche, Nucl. Phys. A713, 231 (2003).

10. A.J. Koning, et al., TALYS-1.0, Proceedings of the International Conference on Nuclear Data for Science and Technology, 22-27 May (2007)

11. C.Kalbach, Phys.Rev., C37, 2350(1989).

12. A.V. Ignatyuk, et al., Sov. J. Nul. Phys. 21, 255 (1975).

13. A. Aydin, J. of Fusion Energy 29(5), 476 (2010).

14. A. Kaplan, J. Fusion Energ. 32 (3), 382 (2013).

15. B. Sarer et al., Ann. Nucl. Energ. 45(36), 417 (2009).

16. T. Belgya, et al., Handbook for Calculations of Nuclear Reaction Data, RIPL-2 (IAEA-Tecdoc1506), Taylor \& Francis, (2006).

17. M. Yigit, J. Fusion Energ 34:1392 (2015)

18. H. Korkut, et al., J. Fusion Energy, 35, 3, 591 (2016).

19. M. Aikawa, et al., Astron. Astrophys. 441, 1195, (2005).

20. E.Tel, A.Kara, J. Fusion Energy, 31, 3, 257 (2012).

21. Brookhaven National Laboratory, National Nuclear Data Center, EXFOR/CSISRS (Experimental Nuclear Reaction Data File) Database Version of November 24 (2008). 